\title{
Bacteriological study of Orofacial Space Infections and their Antibiotic Sensitivity Profile
}

\author{
Parul Garg ${ }^{1}$, Varun Goel ${ }^{2 *}$, Jyoti M. Nagmoti ${ }^{3}$ and Mahantesh B. Nagamoti ${ }^{3}$ \\ ${ }^{1}$ Department of Microbiology, National Institute of Tuberculosis and Respiratory Diseases, \\ New Delhi -110030, India \\ ${ }^{2}$ Department of Microbiology, Santosh Medical College and hospital, Ghaziabad, U.P., India \\ ${ }^{3}$ Department of Microbiology, JN Medical College, KLE University, Belagavi, Karnataka, India \\ *Corresponding author
}

\begin{abstract}
Keywords
Odontogenic infection, Submandibular space, Maxillofacial infection Orofacial Space Infections.

Article Info

Accepted:

10 September 2017 Available Online:

10 November 2017 abscess. The purpose of this study was to identify and perform antibiotic susceptibility pattern of aerobes and anaerobes isolates from oral and maxillofacial infections. Fifty patients with space infection of odontogenic origin were selected irrespective of their age and gender. Pus samples were collected and processed in the microbiology laboratory for the growth of anaerobic and aerobic bacteria and antibiotic sensitivity profile. Demographic profile of the patients showed that male patients were more commonly involved and most patients fell into the third and fourth decade of age groups. Submandibular space was most commonly involved in $46 \%$ followed by buccal space in $20 \%$ cases. Staphylococcus aureus was the frequent aerobic bacterial isolate and among anaerobes, Peptostreptococcus spp. was most common 19(44\%) followed by Porphyromonas gingivalis 7(16.27\%). Clindamycin, Gentamycin, Linezolid, Imipenem were the most effective antibiotics. $20 \%$ of the aerobes were resistant to penicillin. There is the predominance of Gram positive bacteria and sensitivity patterns were almost the same reflecting the relevance of ciprofloxacin, ceftazidime and amoxiclav in the treatment of maxillofacial infections. Metronidazole and clindamycin are effective antibiotic to treat anaerobic infection and should be given in combination to cover aerobic bacteria.
\end{abstract}

A B S T R A C T

Orofacial space infections are among the most commonly encountered problems in dental practice. Spreading odontogenic infections are the most common type of serious oral and maxillofacial infections and range from the periapical abscess to superficial and deep neck

\section{Introduction}

Orofacial space infection is most prevalent disease worldwide and is principle reason for seeking dental care. In health, oral microorganisms and human immune system are in ecological balance which is a pressure for sustaining a barrier against ingested pathogenic organism. These infections are mostly of odontogenic origin, as a sequel to pulp necrosis due to caries, trauma, periodontal infections and pericoronitis because some of these infections resolve with little consequences, while some may spread to facial spaces adjacent to the oral cavity and spread aggressively leading to more severe infections. [1, 2] If these infections are ignored or not treated properly, complications 
such as airway obstruction, infection of carotid sheath, meningitis, septicemia, cavernous sinus thrombosis, mediastinitis and distant metastatic foci have been reported. [3, 4]. In the everyday practice of oral and maxillofacial surgeons, empirical antibiotics are prescribed in the face of uncertainty which requires the knowledge of likely pathogenic microbes that may cause orofacial infections and their antibiotic susceptibility pattern in the confined environment as a guide for a rational choice of antibiotic therapy.

Therefore this study was decided to carry out to re-evaluate the presumed pathogens involved in oral and maxillofacial infections and their susceptibility to the routinely used antibiotics in this part of the world.

\section{Materials and Methods}

The present study was conducted at the Department of Microbiology at a tertiary care hospital. Fifty patients presenting with signs and symptoms of abscess of Oral and maxillofacial infections reporting to the department of Oral and Maxillofacial Surgery, over a period of one year from Jan 2012 to Dec 2012 were included in this study. All the patients of oro-maxillofacial infection with clinical evidence of abscess formation and not on antibiotics 48 hours prior to sampling were included.

The pus from the abscess was collected aseptically with the help of syringe and needle and when the abscess is deep and the site is difficult to reach sterile cotton swab was used to collect the sample. After collection, the sample was immediately transferred to thioglycollate broth. Direct smear was made by using a loop full of sample. The smear was stained by Gram's method [Hucker's modification] for an immediate presumptive diagnosis of the number and type of microorganisms present in the sample.
The culture was done for both aerobic and anaerobic bacteria. For aerobic culture sample was inoculated onto 5\% Sheep blood agar, Chocolate agar and McConkey's agar. All the isolates were identified and characterised biochemically by standard procedures. [5] The antimicrobial susceptibility testing was done for aerobic isolates by disc diffusion method as described by Kirby and Bauer for Amikacin (30 mcg), Ampicillin (10mcg), Carbenicillin (100mcg), Ciprofloxacin (5 mcg), Amoxicillin / clavulanic acid (30 mcg), Ceftazidime (30 mcg), Erythromycin (15mcg), Cephoxitin (30mcg),CoTrimoxazole $(1.25 / 23.75 \mathrm{mcg} 0$, Vancomycin (30mcg). Control stain used was Staphylococcus aureus ATCC 25923.

For culture for anaerobic organisms, [6] each sample was inoculated onto Blood agar supplemented with Haemin $(5 \mathrm{mcg} / \mathrm{ml})$ and Vit $\mathrm{K}(10 \mathrm{mcg} / \mathrm{ml})$. Blood agar plates used for anaerobic isolation were prepared with Brucella agar base. Inoculated Chocolate agar plate incubated in a candle jar at $37{ }^{\circ} \mathrm{C}$ to test for aerotolerance.

If there was no growth on plates after 72 hours of anaerobic incubation, plates were reincubated for an additional period of 48 hours. Method used for obtaining anaerobiosis in the jar was "internal gas generating system" described by Lakshminarayana and Vaidhyalingam. [7] Cold catalyst consist of pellets of alumina coated with finely divided Palladium (Baker platinum Ltd., London) that is reactivated every time before use by drying at $150^{\circ} \mathrm{C}$ $160^{\circ} \mathrm{C}$ for $1-2 \mathrm{hrs}$ was used.

Susceptibility to Metronidazole and clindamycin was done by agar dilution method. Bacteroides fragilis ATCC 25285 was used as a control strain. MIC of this strain for clindamycin is $0.5-2 \mu / \mathrm{ml}$ and for metronidazole is $0.25-1 \mu / \mathrm{ml}$. 


\section{Results and Discussion}

A total of 50 samples were collected from patients presenting with maxillofacial abscess from January to December 2012. The ages of the study population ranged between 5 and 100 years with male to female ratio of 1:1.94. Maximum numbers of cases were in the age group of 31-50 years and minimum number of cases seen in 1-10 years.

In the present study the submandibular space was the most common space involved $23(46 \%)$ in maxillofacial infections. The least common space involved was canine space 2(4\%) (Figure 1). While multiple space involvement was seen in 5(10\%) of cases. Pus samples from all 50 cases yielded growth on culture. A total number of 97 isolates were obtained from 50 samples. Polymicrobial growth was present in $36(72 \%)$ samples. Out of total 14 monomicrobial samples, 8 (57.14\%) samples had shown only anaerobic isolates (Figure 2). In 27 (75\%) samples there was mixed infection by both aerobes and anaerobes. Out of these 27 samples, $17(62.9 \%)$ samples had two isolates and $10(27.03 \%)$ samples had three isolates. In only 1 case four isolates was present.

Out of total 50 samples, 54 aerobic organisms were isolated. Gram positive cocci were in 43(75.9\%) samples and gram negative bacilli were $13(24.07 \%)$. Among gram positive isolates Staphylococcus aureus was the commonest organism isolated followed by Streptococcus pyogens. Among these facultative anaerobes $S$. aureus (8), Streptococcus pyogens (6) and Streptococcus viridians (4) were common species found in synergism with obligate anerobes (Table 1). Klebsiella pneumonia was the commonest gram negative bacilli isolated 5(38.4\%) followed by Pseudomonas aeruginosa $3(23 \%)$. The most common anaerobic organism was Peptostreptococcus spp
(25.58\%). Synergy was most commonly found in Peptostreptococcus spp. (10), P. gingivalis (6) and Fusobacterium (3) with aerobic bacteria.

Staphylococcus aureus has shown high resistance to Ampicillin (68.75\%) and Amoxicillin (43.75\%). Staphylococcus aureus isolates had a high sensitivity to third generation cephalosporin ceftazidime $(68.75 \%)$ and ciprofloxacin (81.25\%). Results showed very low sensitivity to macrolide group. Only $43.1 \%$ gram positive cocci were found sensitive to Erythromycin. 70.3\% of Gram negative bacteria was found to be sensitive for ampicillin. Gram negative bacteria had higher sensitivity $(84.6 \%)$ to ceftazidime than to cefuroxime in $46.1 \%$ isolates. Only 1 isolate of $P$. aeruginosa was resistant to Piperacillin-tazobactam. Gram negative isolates had higher sensitivity to Amikacin and Amoxyclav.

In the present study none of the isolates was found to be resistant to metronidazole (Table 2). Most of the isolates were sensitive at MIC of range $0.5 \quad-2 \mu \mathrm{g} / \mathrm{ml}$. 2 isolates of Fusobacterium spp and Peptostreptococcus anaerobicus had higher MIC $4 \mu \mathrm{g} / \mathrm{ml}$. In the present study $28(65.11 \%)$ anaerobic isolates were sensitive to clindamycin while susceptibility of $9(20.9 \%)$ isolates was intermediate. Only 6(13.9\%) isolates were resistant to clindamycin (Table 3). Among the resistant isolates Bacteroides fragilis (33.33\%) followed by Peptostreptococcus spp. (15.79\%) and Porphyromonas gingivalis (20\%). Resistance was not found in Fusobacterium spp. and Prevotella spp.

Maxillofacial infections are a public health concern, mainly related with the odontogenic origin if ignored or ill-treated at the early stage can rapidly develop and spread to neighbouring anatomic structures, leading serious complications like airway obstruction, 
mediastinitis, septicemia, cavernous sinus thrombosis, jugular vein thrombosis, carotid aneurysm and shock. So prevention and prompt management are necessary for countries like India where healthcare providers are inadequate in number and facilities are less. Incision and drainage are the prime treatment for sure, but the understanding of involved bacteriology and sensitivity pattern constitutes an important part of it. [8] Many times even after proper surgical treatment patient condition fails to improve, one of the important reasons for this is resistant bacterial strains and selection of wrong antibiotics.

Table.1 Culture of anaerobic isolates

\begin{tabular}{|l|c|c|}
\hline \multicolumn{1}{|c|}{ Species } & Number $\mathbf{( n = 4 3 )}$ & Percentage $\%$ \\
\hline Peptostreptococcus anaerobicus & 11 & 25.58 \\
\hline Peptococcus saccharolyticus & 8 & 18.6 \\
\hline Porphyromonas gingivalis & 7 & 16.27 \\
\hline Bacteroides fragilis & 6 & 13.9 \\
\hline Fusobacterium spp & 5 & 11.62 \\
\hline Prevotella intermedia & 4 & 9.30 \\
\hline Prevotella melaninogenica & 2 & 4.65 \\
\hline
\end{tabular}

Table.2 Susceptibility pattern of metronidazole

\begin{tabular}{|l|c|c|c|}
\hline \multicolumn{1}{|c|}{ Organism } & $\begin{array}{c}\text { Sensitive } \\
\mathbf{8} \boldsymbol{\mu g} / \mathbf{m l}\end{array}$ & $\begin{array}{c}\text { Intermediate } \\
\mathbf{1 6} \boldsymbol{\mu g} / \mathbf{m l}\end{array}$ & $\begin{array}{c}\text { Resistant } \\
\mathbf{3 2} \boldsymbol{\mu g} / \mathbf{m l}\end{array}$ \\
\hline Peptostreptococcus anaerobicus $n=11$ & 11 & 0 & 0 \\
\hline Peptococcus saccharolyticus $n=8$ & 8 & 0 & 0 \\
\hline Porphyromonas gingivalis $n=7$ & 7 & 0 & 0 \\
\hline Bacteroides fragilis $n=6$ & 6 & 0 & 0 \\
\hline Fusobacterium spp. $n=5$ & 5 & 0 & 0 \\
\hline Prevotella intermedia $n=4$ & 4 & 0 & 0 \\
\hline Prevotella melaninogenica $n=2$ & 2 & 0 & 0 \\
\hline
\end{tabular}

Table.3 Susceptibility pattern of clindamycin

\begin{tabular}{|c|c|c|c|}
\hline Organism & $\begin{array}{c}\text { Sensitive } \\
2 \mu \mathrm{g} / \mathrm{ml}\end{array}$ & $\begin{array}{c}\text { Intermediate } \\
4 \mu \mathrm{g} / \mathrm{ml}\end{array}$ & $\begin{array}{c}\text { Resistant } \\
8 \mu \mathrm{g} / \mathrm{ml}\end{array}$ \\
\hline Peptostreptococcus anaerobicus $n=11$ & 7 & 2 & 2 \\
\hline Peptococcus saccharolyticus $n=8$ & 5 & 2 & 1 \\
\hline Porphyromonas gingivalis $n=7$ & 5 & 1 & 1 \\
\hline Bacteroides fragilis $n=6$ & 3 & 1 & 2 \\
\hline Fusobacterium spp. $n=5$ & 4 & 1 & 0 \\
\hline Prevotella intermedia $n=4$ & 3 & 1 & 0 \\
\hline Prevotella melaninogenica $n=2$ & 1 & 1 & 0 \\
\hline
\end{tabular}


Fig.1 Distribution of cases according to maxillofacial space involved

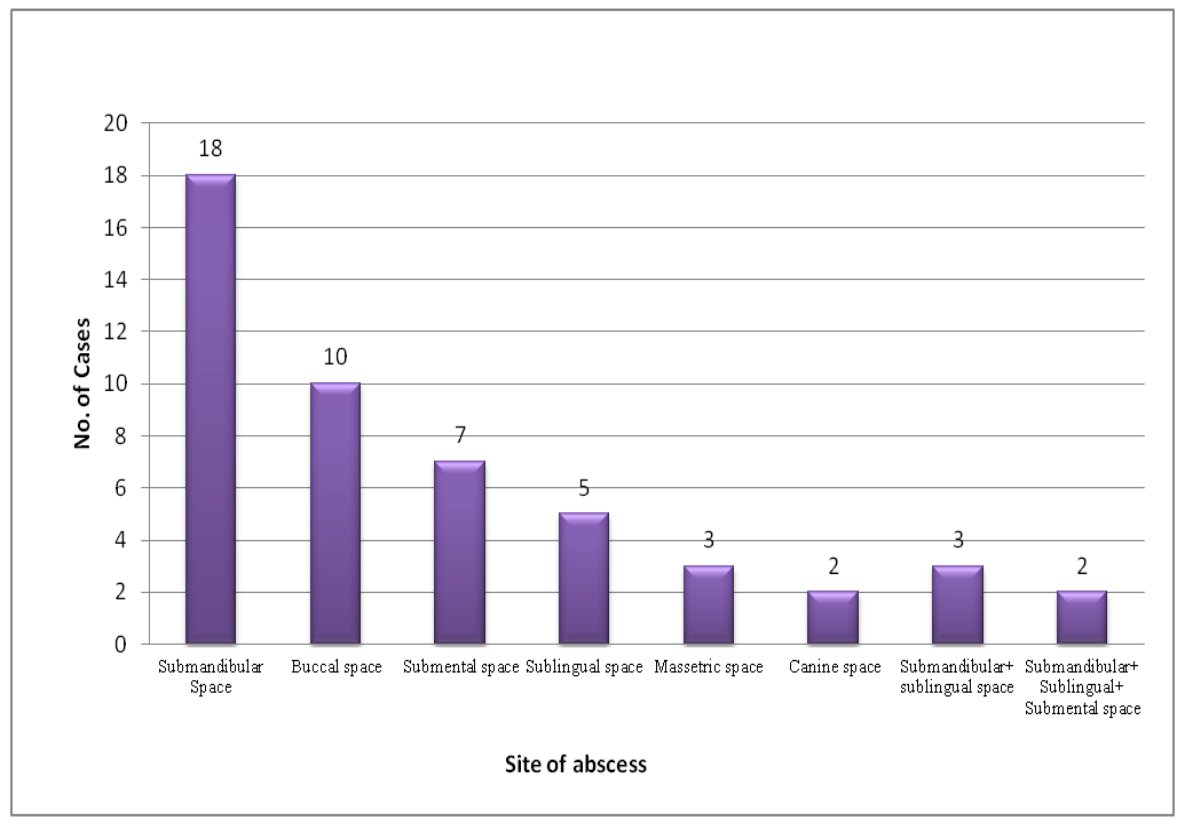

Fig.2 Distribution of type of isolates

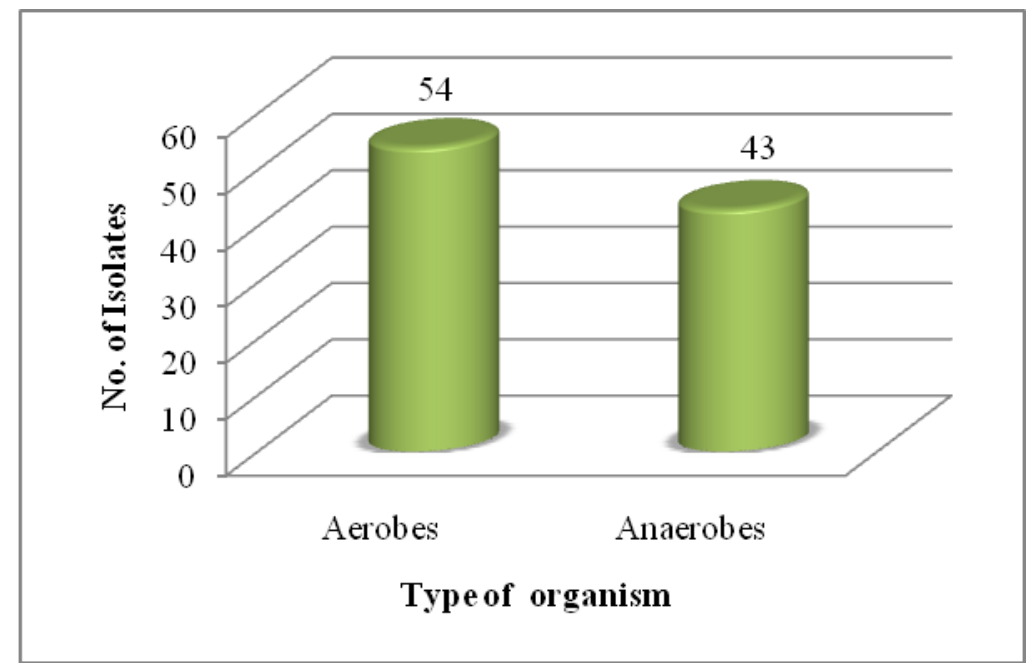

In the present study, the age of patients varied from 5 to 70 years with mean age 33.77 years. Reja et al., studied [9] patients with facial space infections with an age range of 7 to 93 years with mean age of 33.3 years. While Lee et al., found these infections were in the age range of 1 to 89 years with mean age of 33.4 years. [10] In another study by the Suehara et al., mean age of facial space infections was 37.6 years. [11] Our studies correlate well with the findings of these studies. Most of the patients24 (48\%) in our study were adults in the age group of $31-50$ years. This confirms the reports of previous studies that although children may acquire maxillofacial infections $(10 \%)$, the majority occurs in adults in this age group. The probable reason for adults being at higher risk is the neglect of oral health and the higher prevalence of systemic diseases that compromise immunity. Children 
were found to have relatively less occurrence of infection of the primary facial spaces because the erupting permanent teeth resorb their roots making their length short. That is why primary teeth usually present odontogenic infection in the form of a gum boil rather than spreading to the fascial spaces.

In the present study submandibular space was most commonly involved space infected in $23(46 \%)$ cases followed by buccal space in 10 (20\%). Multi-space involvement was present in $5(10 \%)$ of the cases in which submandibular space was the comment as found in single space involvement. Reja et al., in his study reported that submandibular space was involved in $30 \%$ cases followed by buccal space which was involved in $27.5 \%$ cases. [9] A different pattern was observed by Bridgeman et al., where buccal space (52.6\%) was the most common space followed by submandibular space (24\%) in the study. [12] Maxillofacial infections are considered to be polymicrobial in nature caused by both aerobes and anaerobes. [12, 13] Our study also showed polymicrobial infections in 36 $(72 \%)$ of cases as compared to monomicrobial in $14(28 \%)$ cases. Among 14 (28\%) monomicrobial isolates, $16 \%$ pus samples yielded only anaerobic bacteria and $12 \%$ only aerobic bacteria. Among 36 polymicrobial samples, 27 samples showed mixed infection caused by both aerobes and anaerobes. While synergy between two anaerobes and two aerobes was found in $8 \%$ and $10 \%$ of cases respectively. Earlier studies from other parts of the world have reported about mixed anaerobic flora in orofacial infections as found in our study. [2, 14]

According to the literature available, aerobic predominance was seen initially which later on turned towards anaerobes. $[15,16]$ Results by various studies are contradictory till today. In our study, aerobic isolates were $55.67 \%$ and anaerobes $44.32 \%$. A recent study by demonstrated that aerobic organisms outnumbered anaerobes by almost 2:1 ratio. [9] These results were contradictory to many studies who showed anaerobic predominance in the study. [14, 16, 17, 18, 19]

Our study showed out of 54 aerobes isolates, $75.9 \%$ Gram positive cocci were present in pus samples. Looking at aerobic population it was found that Staphylococcus aureus was the most commonly 39\% isolated species followed by Streptococcus pyrogens $21.9 \%$. Staphylococcus aureus is not a normal flora of oral cavity and may be an important pathogenic organism in sappurative nonodontogenic infection of the maxillofacial region. In contrast to our study, multiple studies demonstrated Streptococcus viridans as their predominant species. [20, 21]

Isolation rate of anaerobes was $44.32 \%$ from pus samples in our study. The detection rate of anaerobes from patients with deep space infections was relatively lower than that of observed by, Kuriyama et al., (2000), Yuvraj (2004), Ndukwe (2004), Pathak (2012) and Osazuwa (2010). [13, 14, 17, 19, 22] The isolation of anaerobic bacteria was less in the study could be because they are fastidious in nature, anaerobes are hard to isolate and are often not recovered from the infected sites. However, the different studies have shown $30-90 \%$ isolation rate of anaerobes in their studies. [2, 9, 14, 23] Thus our study shows the coinciding results demonstrating isolation rate of $44.3 \%$. Mixed infection was found in $27(63 \%)$ cases involving both aerobes and anaerobes. Many investigators have demonstrated the mixed infections of Streptococcus spp, Peptostreptococcus, Prevotella, Porphyromonas, and Fusobacterium which were frequently isolated from orofacial odontogenic infections. [19, 23, 24] We have also found synergism most commonly in anaerobic 
isolates of Peptostreptococcus spp (10), Porphyromonas (6) and Fusobacterium (3) with aerobic bacteria. The interactions of organisms within the microbial mixtures leads to a production of virulence factors such as hemolysins, proteases and collagenases that cause inflammation, healing and contribute to the chronicity of infection. In the study, $16 \%$ pus samples yielded pure anaerobic isolates. In the abscess, this pure anaerobic organisms produce in the late stage of abscess formation. [24]

In our study, none of the isolates was found to be resistant to metronidazole. Most of the isolates were sensitive at MIC of range 0.5 -2 $\mu \mathrm{g} / \mathrm{ml}$. Two isolates of Fusobacterium spp and one isolate of Bacteroides fragilis had higher MIC of $4 \mu \mathrm{g} / \mathrm{ml}$.

Time to time analysis of bacterial strains and resistance pattern should be a continuous process so that we do not lag behind at the latest changes. Our study showed that the microbiological flora of orofacial abscess consists of the complex mixture of aerobic and anaerobic bacteria and polymicrobial in nature. Gram positive cocci were more common in aerobic isolates while in anaerobic isolates gram-negative bacilli were common. Most commonly isolated organisms were Peptostreptococcus and Staphylococcus aureus in anaerobes and aerobes respectively. Metronidazole and clindamycin are still effective to treat anaerobic infections. The study clearly indicated when orofacial odontogenic infections are treated with antibiotics, an antimicrobial spectrum against both aerobes and anaerobes may be required.

\section{References}

Bridgeman A, Wiesenfeld D, Hellyar A, Sheldon W. Major Maxillofacial infections: An evaluation of 107 cases. Aust Dent J 1995; 40: 281-8.
Chunduri NS, Madasu K, Goteki VR, Karpe $\mathrm{T}$, Reddy $\mathrm{H}$. Evaluation of bacterial spectrum of orofacial infections and their antibiotic susceptibility. Ann Maxillofac Surg. 2012;2(1):46-50.

Collee JG, Duguid JP, Fraser AG, Marmion BP, Simmons A. (Eds). Mackie and Mc Cartney Practical Medical Microbiology, 14th Edn, Singapore: Churchill Livingstone 1989

Flynn TR, Shanti RM, Levi MH. Severe odontogenic infections, part 1: Prospective report and part 2: Prospective outcomes study. J Oral Maxillofac Surg 2006; 64: 1093-113.

Goldberg $\mathrm{MH}$. The changing biologic nature of acute dental infection. JADA 1970;80:1048.

Greenberg RN, James RB, Marier RL. Microbiologic and antibiotic aspects of infections in the oral and maxillofacial region. J Oral Surg 1979;37:873-84.

Huang TT, Tseng FV, Yeh TH, Hsu CJ, Chen YS. Factors affecting the bacteriology of deep neck infection: A retrospective study of 128 patients. Acta Otolaryngol 2006; 126:396-401.

Kohli M, Mathur A, Kohli M, Siddiqui SR. In Vitro evaluation of microbiological flora of orofacial infections. J Maxillofac Oral Surg 2009;8(4):329_ 33.

Kuriyama T, Karasawa T, Nakagawa K. Bacteriology and antimicrobial susceptibility of gram-positive cocci isolated form pus specimens of orofacial odontogenic infections. Oral Microbiol Immunol 2002; 17(2):132-5.

Labriola DJ, Mascaro J, Alpert B. The microbiologic flora of orofacial abscesses. J Oral Maxillofac Surg 1983; 41: 711-4.

Lee JK, Kim HD, Lim SC. Predisposing factors of complicated deep neck infection: An analysis of 158 cases. Younsei Med J 2007; 48: 55-62. 
Moenning JE, Nelson CL, Kohler RB. The microbiology and chemotherapy of odontogenic infection. J Oral Maxillofac Surg 1989;7:976-85.

Ndukwe KC, Okeke IN, Akinwande JA, Aboderin AO, Lamikrah A. Bacteriology and antimicrobial susceptibility profile of agents of orofacial infections in Nigerians. Afr J Clin Exp Microbial 2004; 5: 272-7.

Osazuwa F, Adebayo AO, Alli OAT, Osazuwa EO. Bacteriology Of Orofacial Infections In Gombe, Nigeria. Academia Arena 2010;2(12):82-4.

Panagiotis K, Stefanopoulos DDS, Alexandros E. The clinical significance of anaerobic bacteria in acute orofacial odontogenic infections. Oral Surg Oral Med Oral Pathol Oral Radiol Endod 2004;98:398-408.

Pathak A, Gupta MK, Pillai A. Incidence of Anaerobic Bacteria in 118 Patients with Deep-space Head and Neck Infections from the People's University Hospital of Maxillofacial Surgery, Bhopal, India. J Orofac Res 2012;2(3):121-6.

Reja AJ, Shahid RA, Ziccardi VB. Microbiology and sensitivity of Head and neck space infections of odontogenic origin. J Oral Maxillofac Surg 2006;64:1377-80.

Simpson AJH. Rational antibiotic therapy. Surgery 2002;20(8):177-9.

Storoe W, Haig RH, Lillich TT. The changing face of the odontogenic infections. J Oral Maxillofac Surg 2001;59:739-48.

Sueharal AB, Gonçalves AJ, Alcadipani FAMC, Kavabata NK, Menezes MB. Deep neck infection: Analysis of 80 cases. Rev Bras Otorhinolaringol 2008; 74: 253-9.

Sutter VL, Citron DM, Edelstein M, Finegold SM. Wadsworth Anaerobic Bacteriology Manual. 4th edition. Star publishing company, California. 1985

Topazian RG, Goldberg MH, Hupp JR. Oral $\&$ Maxillofacial Infections. $4^{\text {th }}$ edition. W.B. Saunders company. 2002.

Vaidhyalingam K, Laxminarayana CS. Internal Gas Generator System suitable for creating Anaerobiosis. Ind J Surg 1980;42:154-9.

Yuvraj V, Alexander M. Is the microflora of Oral \& Maxillofacial infections changing? Int J Oral Maxillofac Surg 2007; 8(285): 1040.

\section{How to cite this article:}

Parul Garg, Varun Goel, Jyoti M. Nagmoti and Mahantesh B. Nagamoti. 2017. Bacteriological study of Orofacial Space Infections and their Antibiotic Sensitivity Profile. Int.J.Curr.Microbiol.App.Sci. 6(11): 1079-1086. doi: https://doi.org/10.20546/ijcmas.2017.611.127 